\title{
Generalized phenomenological equation of plant growth
}

\author{
Sylwia Lewicka ${ }^{1}$ and Mariusz Pietruszka ${ }^{2}$ \\ ${ }^{1}$ The H. Niewodniczanski Institute of Nuclear Physics, Polish Academy of Sciences, Radzikowskiego 152, 31342 Kraków, \\ Poland; E-mail: sylwia.lewicka@ifj.edu.pl \\ ${ }^{2}$ Faculty of Biology and Environmental Protection, University of Silesia, Jagiellońska 28, 40032 Katowice, Poland
}

\begin{abstract}
Enlargement is one of the most fundamental activities of plants, and there are many simultaneous processes involved. Several could be temperature-dependent, like metabolic processes and cell wall sensitivity. A description of plant cell elongation was elaborated by Lockhart in the mid-60's of the last century in the form of time-dependent differential equation. However, the main disadvantage of this approach was the missing environmental temperature at which growth takes place, as well as the lack of representation of environmental factors influencing growth, like growth stimulators/inhibitors, external pressure or light. This absence has been merely covered in the series of our recent papers. Consequently, this manuscript attempts to construct a fairly complete, all-encompassing set of mathematical relationships which describe the basic process of cell/organ extension and the effects of modifying environmental perturbations. The output is provided in a form of composite equation supported by mathematical derivations. Starting with the generalized time- and temperature-dependent growth equation, we involve the action of phytohormones and toxic compounds, and especially of light (photocontrol of plant growth) onto the growth processes. All these external factors (treated as perturbations) are mapped onto the proper terms of temperature modified equation of growth thus giving a new theoretical tool to verify, interpret and draw conclusions concerning data originating from various kinds of plant-physiological experiments. It seems that this novel approach can provide a starting point for further experimentation especially on the plastic and elastic components of the growth process.
\end{abstract}

Key words: Growth equations - Growth stimulators/inhibitors — Light — Plant development

\section{Introduction}

Plant growth is based on irreversible extension of the whole organism due to the increase of quantity and size of cells, the mass of protoplast and cell walls (Cosgrove 1986, 1993; Kutschera 2000). In the growth of arbitrary organ one can differentiate three basic phases: the initial phase of slow growth, the intense growth phase and, eventually, the final phase of slow growth (Fogg 1975). The dominating length increments in the time unit are observed approximately at the mid time of growth (2-4 day after germination). The period of intense growth lasts fairly short and depends on plant species. The plant growth is influenced by physical (abiotic)

Correspondence to: Mariusz Pietruszka, Faculty of Biology and Environmental Protection, University of Silesia, Jagiellońska 28, 40032 Katowice, Poland

E-mail: mariusz.pietruszka@us.edu.pl and biotic factors of environment (Wright 1966; Cleland 1986; Trewavas 1991; Edelmann 1995). The fundamental modifying environmental perturbations are: temperature, light, water and soil factors, and atmosphere composition. Growth regulators are also of the fundamental importance in growth and development (see also: Cleland 1986, Arteca 1996 and Pietruszka et al. 2007) in the context of the model presented. These substances stimulate or inhibit the processes of growth. The natural growth regulators (plant phytohormones) include auxins (Rayle and Cleland 1992; Bandurski et al. 1995), giberellins (Ross et al. 2003), cytokinins (Nikolić et al. 2006), ABA and jasmonic acid (Montague 1997; Tsai et al. 1997) and ethylene (Madlung et al. 1999; Cao et al. 2007). The main purpose of the series of our recent papers was to introduce the influence of temperature (Lewicka and Pietruszka 2006), unilateral action of light (Pietruszka and Lewicka 2007a) and gravity (Lewicka and Pietruszka 2007), external pressure (Pietruszka and Lewicka 2007b) and action 
of plant stimulators/inhibitors (Pietruszka et al. 2006; 2007) onto Lockhart's equations of growth. In the utilized models temperature entered the modified equation of growth in two ways: by a state equation, which causes modification of the Lockhart term, and by the Lorentz-like dependence of elongation. We recall that the equation of state is a constitutive equation describing the state of the system under a given set of physical conditions. Especially, it provides a mathematical relationship between two or more state functions associated with the matter, such as its temperature, pressure, volume, or internal energy. The problem of anisotropic growth due to unilateral action of external stimuli (gravity and light) has been undertaken in Lewicka and Pietruszka (2007) and Pietruszka and Lewicka (2007a), respectively; however, the role of temperature was omitted there. In both papers, we proposed a generalization of the scalar Lockhart model to the three-dimensional tensor equation. Also, the proposed methods are consistent with the recent investigations concerning specific auxin-influx and -efflux carriers (LAX and PIN proteins) for phototropism (Friml 2003; Reinhardt et al. 2003) and Cholodny-Went theory for gravitropism (Went and Thimann 1937; Scott et al. 1992). The effect of temperature on plant elongation and cell wall extensibility was also studied in Pietruszka and Lewicka (2007b), where the action of external mechanical pressure was involved. There, we combined experimental and theoretical results based on the separation of elastic effects from growth and summarized our results in the form of phase diagram.

One external signal whose perception and transduction have been particularly well studied is light (De Greef 1996). Light is an exciting factor for many living beings. In the very essence of light there exists another dualistic difference - an energy/information one - for the whole realm of the living world. In particular, in the plant kingdom light powers photosynthesis and means information transfer through signal transduction. The information provided by the light environment can be perceived by different plant photoreceptors: phytochromes, cryptochromes and UV-photoreceptors. It is now commonly accepted that the phytochrome family of photoreceptors is primarily responsible for sensing the red and far-red regions of the spectrum. These pigments translate light cues into biochemical signals subsequently transduced through (largely unknown) pathways into molecular and physiological changes that modulate growth and development (Casal et al. 1998; Deng and Quail 1999; Smith 2000). Also plants respond to light through a variety of forms of phototropism (Friml 2003; Reinhardt et al. 2003; Pietruszka and Lewicka 2007a) and photonasty (Koller 1990). Additionally, visible radiation drastically influences the appearance of a plant. In higher plants, red/far red and blue/UV(A,B)-light direct the life cycle. Light also controls development (growth, differentiation, maturation) of higher plants independently of photosynthesis by photomorphogenesis (Christie and
Briggs 2001; Nakasako et al. 2005). It acts as elicitor that plays a decisive role in regulating the process of optimization of a developing plant. Without the light factor normal plant development cannot occur. The fact that the juvenile stem of dicotyledonous seedlings rapidly elongates in darkness and that this process is inhibited upon irradiation with light has long been known (see Kutschera 2000 for a review). Early studies led to hypothesis that radiant energy may cause an inhibition in the rate of elongation. Further experiments revealed that light retards not only the rate of stem elongation but also causes a reduction in the plastic extensibility of the cell walls. Lockhart (1965b) concluded that visible radiation causes an inhibition of stem elongation due to decrease in cell wall extensibility. Thus, the challenge is to formulate a physical model for the emerging mechanisms in plants exposed to the irradiation of light. In this paper we focus our efforts on understanding the biophysical response to the light irradiation by considering a fully modified equation of growth.

In the last few decades, some papers have been written concerning plant growth as only dependent on time variable (Lockhart 1965a,b; Cosgrove 1986, 1993; Proseus et al. 1999; Lewicka 2006) and the problem of external influencing factors, like temperature or light, has been left open to the theoretical analysis. Therefore, the aim of this study is to present a full model able to report not only on time dependencies of the growth but also responses due to physical and biotic factors. Such a model would presumably well reproduce the existing data, as well as anticipate new growth related features, especially, act as a basis for further experimentation, also on the plastic and elastic components of the growth process. Having all these in mind, it was our desire to bring this kind of approach to the attention of potential readers interested in development and growth. We stress that the presented model equations can be useful in the laboratory practice to determine many growth-accompanying quantities.

\section{Materials and Methods}

The experiments were carried out with three-day-old maize plants (Zea mays L.) grown on Hoagland's medium (Hoagland and Arnon 1950) at $27^{\circ} \mathrm{C}$. Seeds of maize were cultivated in the darkness. Then individual seedlings were transferred to an aerated solution containing standard microand macro-elements. We have chosen seedlings of the same coleoptiles' length, i.e. about $2 \mathrm{~cm}$. In both cases, the length of the elongation zone of each coleoptile was measured.

a) Unperturbed experiment (control, dark): The seedlings were divided into eight groups growing at different temperatures $2,10,20,23,26,27.5,32$ and $40^{\circ} \mathrm{C}$. Each group was represented by 10 seedlings. The experiment was carried out 
within 4 hours, and the measurements were taken at every half an hour by the use of the planimetry method.

b) Light perturbed experiment. The preparation of the plant material was the same as in the case of unperturbed experiment, however, the plants were exposed to the sun light. The "light experiment" also lasted for 4 hours. The length of coleoptiles was measured by the standard planimetry method. In both cases ( $\mathrm{a}$ and $\mathrm{b}$ ) the absolute error has been estimated as not exceeding $0.05 \mathrm{~cm}$.

\section{Thermodynamics of irreversible extension of plant cell/organ}

Time-dependent Lockhart equation elaborated in the mid-60's of 20th century (Lockhart 1965a) describing the elongation of a plant cell resulting from a dynamic balance between the water uptake and the cell wall yielding should in principle, along with environmental temperature $T$, include other external factors like growth stimulators/inhibitors and, especially, the influence of light. Hence, as a fundamental differential equation we propose the following model, instead:

$$
\frac{1}{V} \frac{d V}{d t}=\Phi(\underbrace{P-Y}_{\text {Lockhart term }}+\underbrace{p_{1} \theta\left(t-t_{1}\right)}_{\text {stimulator/inhibitor }}-\underbrace{p_{2}}_{\text {light }}+\underbrace{P_{\text {ext }}}_{\text {external pressure }})
$$

where $P$ and $Y$ stand for hydrostatic pressure and turgor threshold, respectively, see Fig. ${ }^{1}$ ). The next two terms describe the action of phytohormones and light which in the framework of our model both act as outer perturbations. The latter term in Eq. (1) characterizes the presence of an external pressure (for full discussion see Pietruszka and Lewicka 2007). In the above equation $V=V(t)$ denotes the cell/organ volume, $p_{1}$ and $p_{2}$ represent inner pressures relevant to the influence of growth stimulators/inhibitors, and to the growth retardation (the minus sign in Eq. (1)) upon irradiation with incident light, respectively. The function $\theta$ stands for the Heaviside theta distribution which is coupled to the stimulator/inhibitor switch at a time $t_{1}$ (i.e. it equals 0 for $t \leq t_{1}$ and 1 for $t>t_{1}$, see also the figure and comment in Pietruszka et al. 2007 (p. 20) for explanation). The righthand side of Eq. (1) is linked to the growth rate by the cell wall extensibility coefficient $\Phi=\Phi(T, t)$. Lockhart equation describes the relative growth as a product of an external

\footnotetext{
1) The method based on making reasonable simplifying assumptions that allows for analytical solutions which yield clear interpretations is always the best to start with. Such methodology which is commonly accepted in science we employ in this article. Moreover, a good theory tends to keep the number of free parameters down to minimum. This way, the consequences of variation of these parameters become transparent. Here we have only two additional parameters $\left(p_{1}, p_{2}\right)$ which, however, can be treated separately by putting one of them equal to zero. This obviously reduce the number of free parameters of our model to indispensable minimum.
}

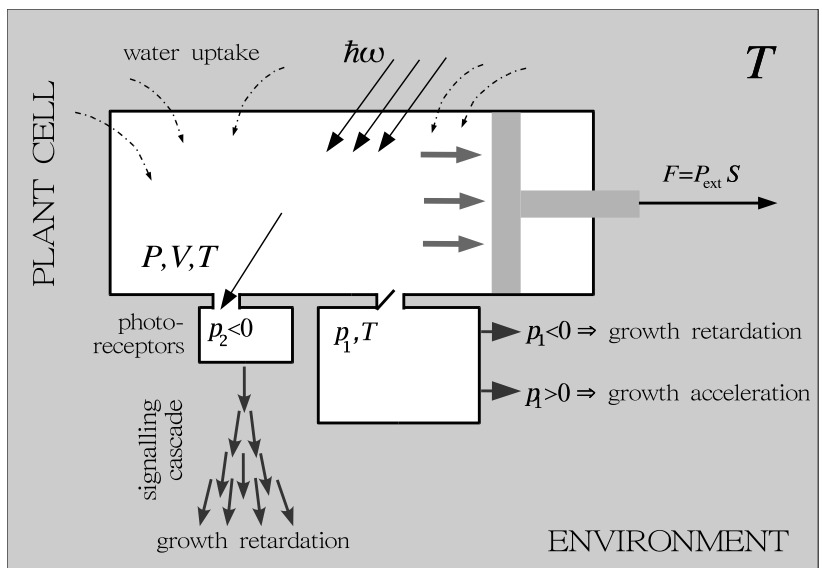

Figure 1. Scheme of the "Gedanken experiment" set-up: Movement of the piston in the cylinder reflects the extensibility properties of a cell wall. A container with pressure $p_{1}$ and a valve which can be opened at a time $t_{0}<t=t_{1}$ is bound with the action of growth stimulator or inhibitor. An additional container with pressure $p_{2}$ represents the (negative) influence of light on the elongation growth (the incident light quanta of energy $\hbar \omega$ are absorbed by photoreceptors inducing signalling pathway of events, which finally leads to growth retardation). The (possible) external mechanical pressure acting on the plant cell is represented by the applied force $F$. The whole system (the plant cell/organ) is immersed into a thermostat (environment) at a temperature $T$.

coefficient $\Phi$ and the driving force. Obviously, stimulator/inhibitor as well as phytochrome-mediated inhibition by light are expected to be present in this equation. Indeed, both are present in the "force term" as well as in the cell wall extensibility coefficient $\Phi$ - the deformation of $\Phi$ enters via the Lorentz-like temperature-dependent elongation as it is shown further in the text and Figs. 4-5. The modified turgor pressure $P$ should be present in this equation in order to introduce possible corrections caused by the action of stimulators/inhibitors or light (water uptake must occur). Nonetheless, the cell wall extensibility also varies with time and temperature. The ensemble of these features reflects the property of time-irreversibility.

In the current model temperature enters the modified equation of growth by three paths: through a state equation (which causes modification of the Lockhart term), by the Lorentz-like dependence of elongation, Planck's distribution and light absorption efficiency in the case of light term, as well. Our proposal is to use such a form of the state equation where the turgor pressure is proportional to the temperature and inversely proportional to the cell/organ volume (we accommodate the argumentation given by Stanley 1971). Such approach is fully justified in first approximation if one begins with the virial theorem and restrict only to the first term of expansion. However, if appropriate assumptions are 
made about the mathematical form of intermolecular forces in the water solution within the vacuole, higher coefficients in the virial expansion should be included. For the sake of clarity and brevity of our model we restrict to the term linear in temperature. Such a choice is also advantageous since it delivers the solution linear in time for the cell/organ volume stretching observed most frequently in growth experiments (e.g. Lewicka and Pietruszka 2006; Pietruszka et al. 2006). Regarding temperature entering the Lorentz-like distribution, see the comments in Pietruszka et al. 2007, pp. 17-18 and 19-20. The third path temperature enters the modified growth equation is via a term bounded with absorption of the light quanta by photoreceptors, which we describe by the Planck's distribution and the efficiency of the process.

Therefore, we use the state equation in a form $P(T, V)$ $Y(T, V)=\gamma T / V$ where $T$ is the absolute temperature, and $V$ is the amount of water in the cell/organ (approx. cell/organ volume). The coefficient $\gamma$ in the first approximation may be treated as constant - in this paper we follow the reasoning concerning the introduction of temperature via the equation of state as in Stanley (1971). Pressure $p_{2}$ can be simply acquired from the energy density $\Delta E / V=W / V=(W / l) / S=$ $F / S=p_{2}$, which in fact is equivalent to the work $W$ done at a distance $l$ by a force $F$ acting onto a surface $S$. Thus the proposed modified Lockhart equation reads

$$
\frac{1}{V(t)} \frac{d V(t)}{d t}=\Phi(T, t)\left(\gamma \frac{T}{V(t)}+p_{1} \theta\left(t-t_{1}\right)-\eta \frac{\Delta E}{V(t)}\right)
$$

and $\eta$ reports on the efficiency of irradiation. In order to reduce the number of free parameters $\eta(T)$ can be obtained by assuming $\eta\left(T_{\text {opt }}\right)=1$ and finding the approximate functional form (see Fig. 3). The total assimilated energy of irradiation $\Delta E$ we introduce by the integral over Planck's distribution

$$
\frac{\Delta E}{V}=\int_{\substack{\text { optical } \\ \text { range }}} \frac{8 \pi h}{c^{3}} \frac{v^{3} d v}{e^{\frac{h v}{k_{B} T}}-1}
$$

where the frequency $v=c / \lambda$, and $\lambda$ is the wave-length of the incident light quanta; $h=2 \pi \hbar$ stands for the Planck's constant. Some further considerations about the coefficient $\eta$ and calculations of $\Delta E$ we have conveyed to the Appendix.

An additional remark needs to be made: even though many global aspects of plant growth have been considered in Eq. (2), the latter is obviously confined to the thermodynamical features. Eq. (2) does not explicitly account for many phenomena taking place in a living cell, like the effect of protein synthesis, cell respiration or concentration of osmotic effectors. Nevertheless, all these effects seem to be swept under the elongation curve (the Lorentz-like distribution). In this sense, and because the experiments are performed on growing coleoptiles of Zea mays L., in our model we are dealing with the phenomenology of a living plant cell growth.
Also the validity of the asymmetric Lorentz-like fit is in fact limited to the temperature region where the Lockhart equation holds and this is surely well below the high temperature edge. Our observations are also in accordance with other literature data: Ikeda et al. (1999) measured growth rates of kidney beans at various temperatures together with water potential, osmotic potential and turgor, and they found that turgor was lost at $40^{\circ} \mathrm{C}$ due to the leaky membrane. Additionally, water uptake related to cell expansion was inhibited at the same temperature. In the work of Nakamura et al. (2002) the similar behavior seemed to be happening at $50^{\circ} \mathrm{C}$. If turgor is lost completely at high temperature, Lockhart equation will not be valid at such temperature any longer. This is the limitation (high-temperature edge limit) of our Lorentz-like model (see also Pietruszka and Lewicka 2007).

\section{Unperturbed case}

In order to solve Eq. (2) for plant cells and organs growing in the darkness and free of any external influence (action of chemical or physical perturbations) we put $p_{1}=0$ and $\Delta E=$ 0 . Hence we obtain its simplified form

$$
\frac{1}{V(t)} \frac{d V(t)}{d t}=\Phi(T, t) \gamma \frac{T}{V(t)}
$$

which, after some calculus, gives the following solution

$$
V(T, t)=V_{0}+\gamma T \underbrace{\int \Phi(T, t) d t}_{\xi_{t}}=V_{0}+\gamma T \xi_{t}
$$

where $V_{0}$ is the cell/organ volume at the beginning of experiment and $T$ denotes the absolute temperature. Here, by denoting $V(T, t)$ we emphasized that the volume also depends on temperature, but to remain the mathematical expressions clear, we only stay at $V(t)$ simultaneously keeping in mind that $V$ also depends on other factors, such as temperature. Since in biological practice we measure temperature in Celsius scale, we rewrite Eq. (5) in the form given beneath

$$
\operatorname{Elong}(\tau, t) \equiv V(\tau, t)-V_{0}=\gamma(\tau+273.15) \xi_{t}
$$

\section{Linear response}

By assuming $p_{1}=b_{1} / V=$ const, we may rewrite Eq. (2) in the form

$$
\begin{aligned}
\frac{1}{V(t)} \frac{d V(t)}{d t} & =\Phi(T, t)\left(\gamma \frac{T}{V(t)}+\frac{b_{1}}{V(t)} \theta\left(t-t_{1}\right)-\eta \frac{\Delta E}{V(t)}\right) \\
& \Downarrow \\
\frac{d V(t)}{d t} & =\Phi(T, t) \overbrace{(\gamma T-\eta \Delta E)}^{t-\text { independent }}+\Phi(T, t) b_{1} \theta\left(t-t_{1}\right)
\end{aligned}
$$

which after integration yields 


$$
V(t)-V_{0}=(\gamma T-\eta \Delta E) \xi_{t}+b_{1} \underbrace{\int \Phi(T, t) \theta\left(t-t_{1}\right) d t}_{I}
$$

Simple considerations and continuity condition lead us to the conclusion that for $t<t_{1} \mathrm{I}=0$ and for $t \geq t_{1} \mathrm{I}=\xi_{t}-\xi_{t_{1}}$ $\left(\xi_{t}=\xi(t)\right.$ denotes the integral of $\Phi(T, t)$ over $\left.t\right)$ which gives

$$
I \equiv \vartheta\left(\xi_{t}-\xi_{t_{1}}\right)=\left\{\begin{array}{cc}
0 & t<t_{1} \\
\xi_{t}-\xi_{t_{1}} & t \geq t_{1}
\end{array}\right.
$$

Hence, the final solution for Eq. (2) in the linear case reads

$$
V(t)=\left\{\begin{array}{cc}
V_{0}+(\gamma T-\eta \Delta E) \xi_{t} & t<t_{1} \\
V_{0}+(\gamma T-\eta \Delta E) \xi_{t}+b_{1} \cdot\left(\xi_{t}-\xi_{t_{1}}\right) & t \geq t_{1}
\end{array}\right.
$$

This linear solution we associate with such a special kind of experiment when the stimulator/inhibitor is added once at a time $t=t_{1}$ (see Pietruszka et al. 2006, 2007). It is worth noticing that above equation includes the unperturbed case if one put both $\Delta E$ and $b_{1}$ equal to zero. Having Eq. (10) and a functional dependence of the volume $V$ on time and temperature, we are able to determine $\Phi(T, t)=\Phi(\tau, t)$ where $\tau$ is the measured temperature (in Celsius scale) and $T=\tau+273.15^{\circ} \mathrm{C}$, in the following manner. For $t \geq t_{1}$ we construct an elongation function as

$$
\begin{aligned}
\operatorname{Elong}(\tau, t) & =V(t)-V_{0} \\
& =(\gamma T-\eta \Delta E) \xi_{t}+b_{1} \cdot\left(\xi_{t}-\xi_{t_{1}}\right) \\
& =(\gamma(\tau+273.15)-\eta \Delta E) \xi_{t}+b_{1} \cdot\left(\xi_{t}-\xi_{t_{1}}\right)
\end{aligned}
$$

On the other hand we define (for justification, see Pietruszka et al. 2007, pp. 19-20)

$$
\operatorname{Elong}(\tau, t)=\frac{\phi_{0}(t) \tau}{\sqrt{\alpha(t)^{2}+\left(\tau-\tau^{*}\right)^{2}}}
$$

where $\phi_{0}$ and $\alpha$ stand for the height and the width of the Lorentz-like curve, respectively; $\tau^{\star}$ denotes the optimum (critical) temperature of growth. Having obtained dependence of the elongation on temperature, we are able to calculate the cell wall yielding $\Phi(\tau, t)$ by comparing Eqs. (11) and (12). This may be accomplished by determining for the fixed times all needed coefficients $\left(\phi_{0}, \alpha\right.$ and $\left.\tau^{*}\right)$ by fitting procedure from the experimental data and by finding their time evolution. The final equation for the cell wall yielding $\Phi(\tau, t)$ (with respect to its initial value at $t_{i}$ for a juvenile cell) in the case of linear perturbation reads

$$
\begin{aligned}
& \Phi(\tau, t)-\Phi\left(\tau, t_{i}\right)=\frac{\tau}{(\gamma(\tau+273.15)-\eta \Delta E)+b_{1}} \times \\
& \times\left(\frac{\phi_{0}{ }^{\prime}}{\sqrt{\alpha^{2}+\left(\tau-\tau^{*}\right)^{2}}}-\frac{\phi_{0} \alpha \alpha^{\prime}}{\left(\alpha^{2}+\left(\tau-\tau^{*}\right)^{2}\right)^{3 / 2}}\right)
\end{aligned}
$$

where the prime denotes the time derivative. The above equation represents the exact form of the analytically derived cell wall yielding coefficient $\Phi(\tau, t)$ in the linear case, with respect to its initial value at $t_{i}$ for the juvenile cell. Wishing to calculate numerically this quantity we need to have $\phi_{0}(\mathrm{t})$, $\alpha(t)$ and $\tau^{*}$ from the fits to the experimental data and the (constant) $\gamma$ value. Calculation of the latter magnitude is accomplished by fulfilling the condition that the measured difference $P-Y$ in plant cells is of the order of 0.1 MPa. In numerical calculations the exact value of $\gamma$ is not that important as it changes only the amplitude (the peak value) of the calculated cell wall yielding coefficient but the order of magnitude still remains the same.

\section{Non-linear response}

By assuming $p_{1}=$ const. we get the final version of Eq. (2) as follows

$$
\frac{d V(t)}{d V}=\Phi(T, t)\left((\gamma T-\eta \Delta E)+p_{1} V(t) \theta\left(t-t_{1}\right)\right)
$$

which is an inhomogeneous differential equation of the first order. It can be solved by standard method in consequence giving

$$
V(t)=\left\{\begin{array}{cl}
V_{0}+(\gamma T-\eta \Delta E) \xi_{t} & t<t_{1} \\
V_{0} e^{p_{1}\left(\xi_{t}-\xi_{1}\right)}+(\gamma T-\eta \Delta E) \xi_{t} & t \geq t
\end{array}\right.
$$

The above non-linear solution we associate with the action of stimulator/inhibitor with concentration increasing in time after $t=t_{1}$ (see Pietruszka et al. 2006, 2007). Next we are able, in analogy to the linear case, to determine the cell wall yielding $\Phi(\tau, t)$

$$
\begin{gathered}
\Phi(\tau, t)-\Phi\left(\tau, t_{i}\right)=\frac{1}{p_{1}} \frac{\tau}{V_{0}-(\gamma(\tau+273.15)-\eta \Delta E) \xi_{t}+\frac{\phi_{0} \tau}{\sqrt{\alpha^{2}+\left(\tau-\tau^{*}\right)^{2}}}} \times \\
\times\left(\frac{\phi_{0}{ }^{\prime}}{\sqrt{\alpha^{2}+\left(\tau-\tau^{*}\right)^{2}}}-\frac{\phi_{0} \alpha \alpha^{\prime}}{\left(\alpha^{2}+\left(\tau-\tau^{*}\right)^{2}\right)^{3 / 2}}\right)
\end{gathered}
$$

The above expression is the final form of the relative cell wall extensibility coefficient (with respect to its initial value at $t_{i}$ for the juvenile cell) $\Phi(\tau, t)$ for the case of non-linear perturbation.

\section{Fitting procedure}

In order to make our paper complete we need to add some comments about the way we have fitted the empirical data and how we have bound them with the theoretical model. In both cases, control and perturbed, based on elongation data, we have obtained distinct sets of coefficients $\phi_{0}, \alpha$ and $\tau^{*}$ via non-linear regression procedure (Levenberg-Marquardt algorithm). 
1. In the "unperturbed case" $\phi_{0}(t)$ increases linearly, while $\alpha(t)$ slightly changes in course of time (nonlinear behavior with wide optimum at about mid-time of growth, hence the polynomial interpolation of the second order), $\tau^{*}$ remains constant.

2. In the "light perturbed case" $\phi_{0}(t)$ and $\alpha(t)$ linearly depend on time, however $\tau^{*}$ decays with time exponentially to the value $22^{\circ} \mathrm{C}$ and tends asymptotically to the $22^{\circ} \mathrm{C}$ line.

Such estimated coefficients have been inserted into Eq. (13) (with $\Delta E=0$ ) in the „unperturbed case“, and Eq. (13) (with $b_{1}=0$ ) in the "light case“, respectively, to receive the theoretically calculated values of $\Phi(\tau, t)$. The calculated determination coefficient $\mathrm{R}^{2}$ in all cases was greater than 0.99 .
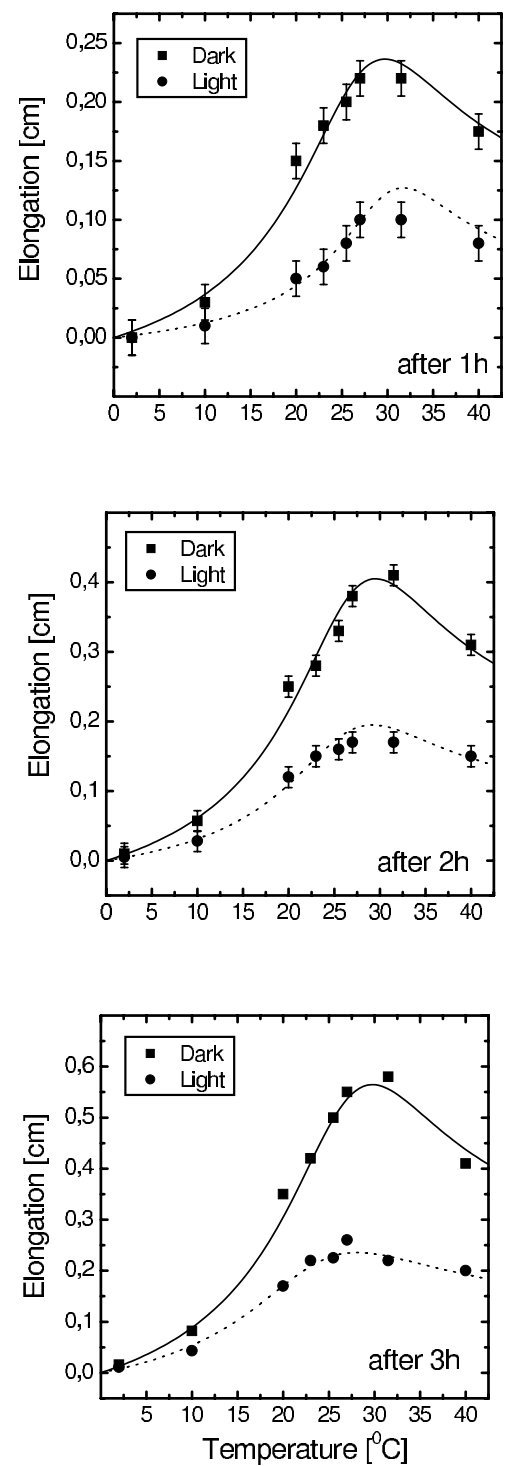

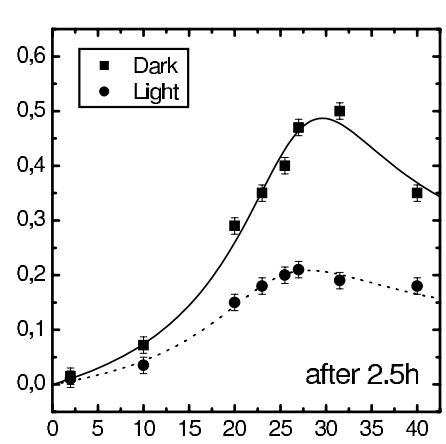

\section{Results}

In this section we consider the main empirical and theoretical results. In Fig. 2 the data from authors' experiments are combined with the semi-theoretical fits. The solid and dotted curves are obtained by the fit to the Lorentz-like distribution, Eq. (12). Fig. 2 presents a sequence of elongation events at fixed times where the measurements are taken both in the darkness and when the seedlings are exposed to the visible light. The empirical data, visualized as points (the error bars are also shown) in Fig. 2, are interpolated by the Lorentzlike curve as defined in Eq. (12). Two main observations are made: in the case of exposure to the light, the strong damping
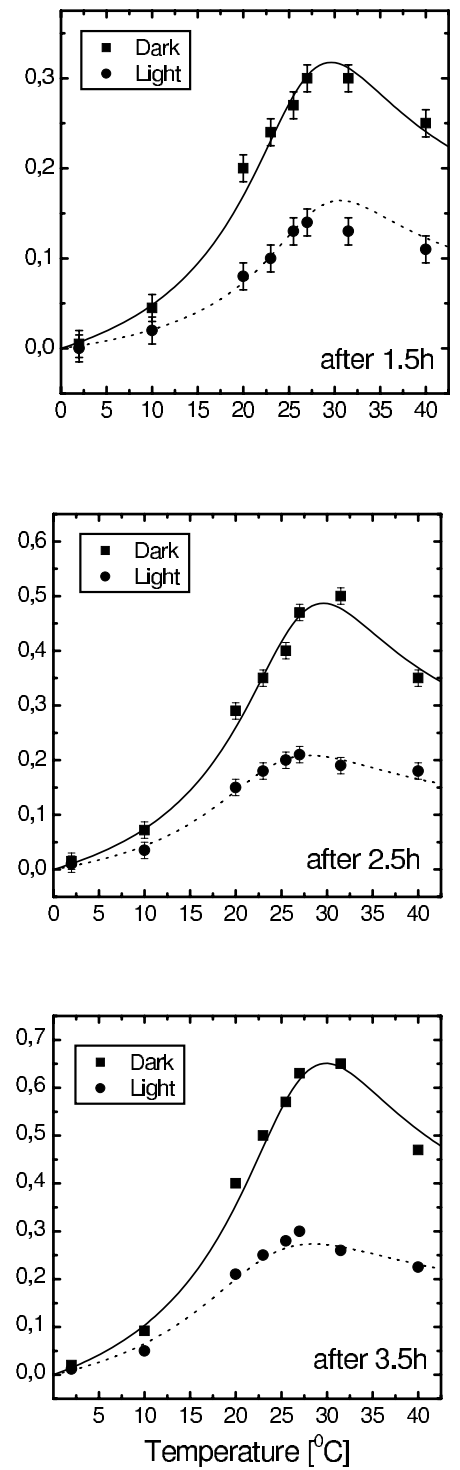

Figure 2. Experimental results: Elongation of maize (Zea mays L.) coleoptiles grown in the darkness (solid line) and in the day light (dotted line) versus temperature for fixed times, fitted by a Lorentz-like distribution. The fitting parameters (Eq. (12)) have been estimated by the non-linear regression procedure (Levenberg-Marquardt algorithm) with determination coefficient $\mathrm{R}^{2} \cong 0.99$. 
with advancing time occurs (flattening of the peak) (a), the displacement of the peak maximum into lower temperatures appears (b). Taking also into account our empirical results as depicted in Fig. 3 we can make an interesting observation. The strongest light-induced growth damping is localized in the close vicinity of its optimum temperature. Even though the biochemistry is not discussed in this paper (or rather it is implicit in the model equations) the observed peak in the dark/light ratio (Fig. 3) should be accounted for the temperature dependencies of enzymatic activity and protein denaturation.

Fig. 4. presents contour plots of the cell wall extensibility coefficient $\Phi(\tau, t)$, as calculated by our model for elongation of maize coleoptile segments linearly perturbed by single application of growth regulators (IAA, $\mathrm{CdCl}_{2}$ ) or non-linearly perturbed by multiple application of the substances in

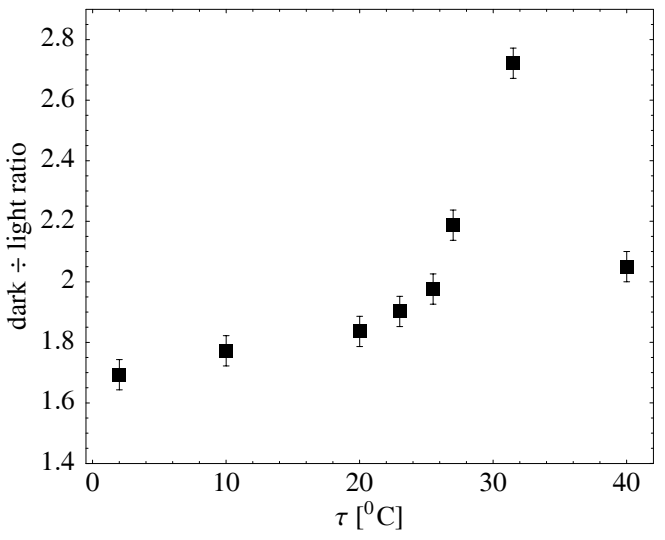

Figure 3. The empirical values of growth (elongation in dark $\div$ elongation in light) ratio in function of temperature. The relative error has been estimated by the logarithmic method.
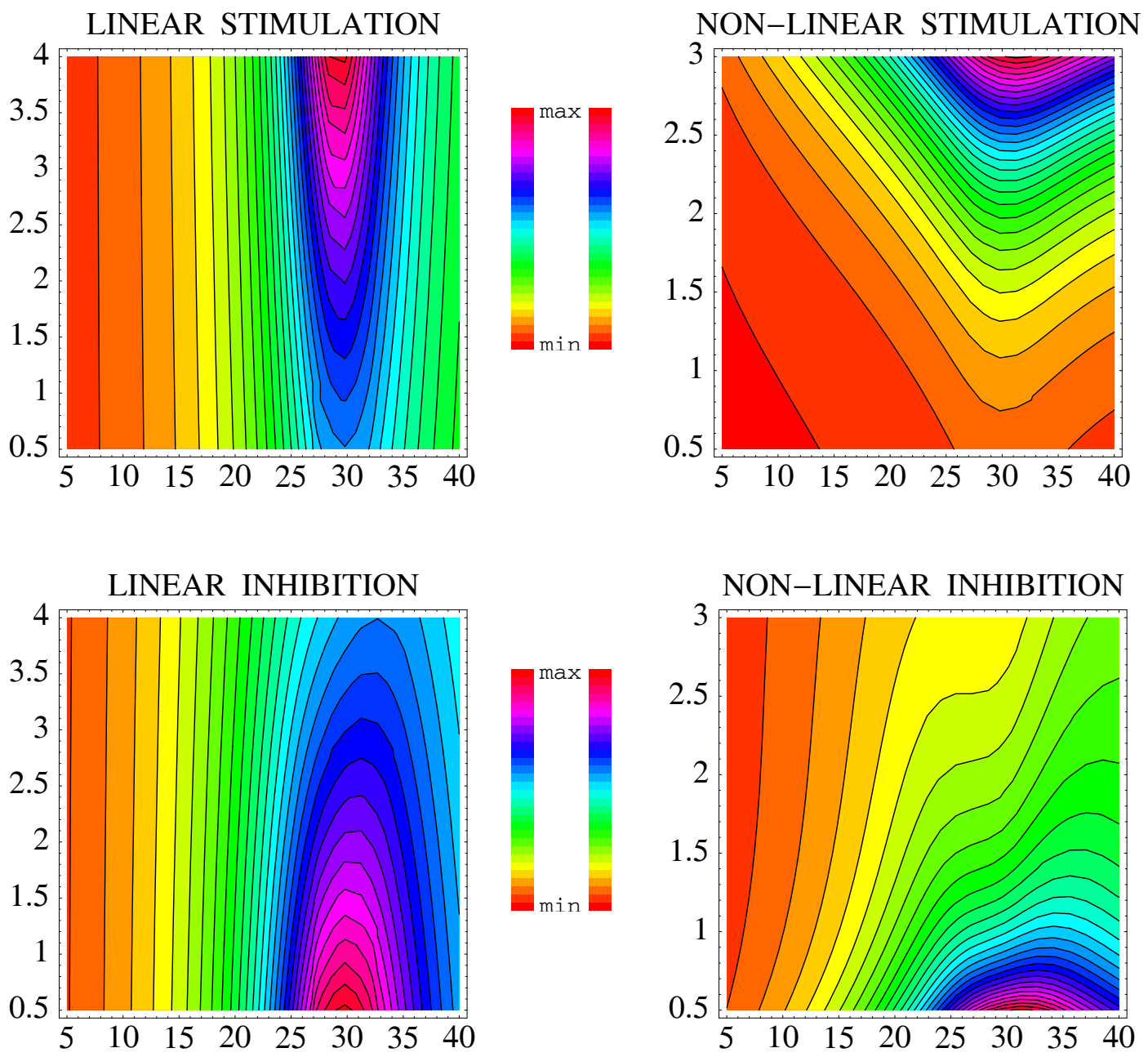

Figure 4. Contour plots (theory) of the calculated cell wall extensibility coefficient $\Phi(\tau, \mathrm{t})\left[\mathrm{cm}^{3} \mathrm{~J}^{-1} \mathrm{~h}^{-1}\right]$ for maize coleoptile segments growing at different environmental conditions: growth stimulation by indole-3-acetic acid or inhibition by cadmium dichloride (in the cases of linear or non-linear response). Horizontal axis - temperature $\tau\left({ }^{\circ} \mathrm{C}\right)$, vertical axis - time $t(\mathrm{~h})$. 
increasing concentrations (see Pietruszka et al. 2007). The upper left plot presents the response of $\Phi$ to the action of the stimulator which causes a moderate linear increase of $\Phi$ in function of time. Moreover we observe a narrowing of the crest at the optimum temperature. Meanwhile, an addition of the inhibitor causes a stronger damping of $\Phi$ with regard to the control (see the lower left plot in Fig. 4 and compare with Fig. 5). The right upper and lower contour plots in Fig. 4 represent results of our model calculations for $\Phi(\tau, t)$ (through Eq. (16)) in the non-linear case. The absolutely different behavior has been found. Constantly added stimulator give raise to the steep exponential increase of $\Phi$ and widening of the hill along the time axis whereas action of the inhibitor
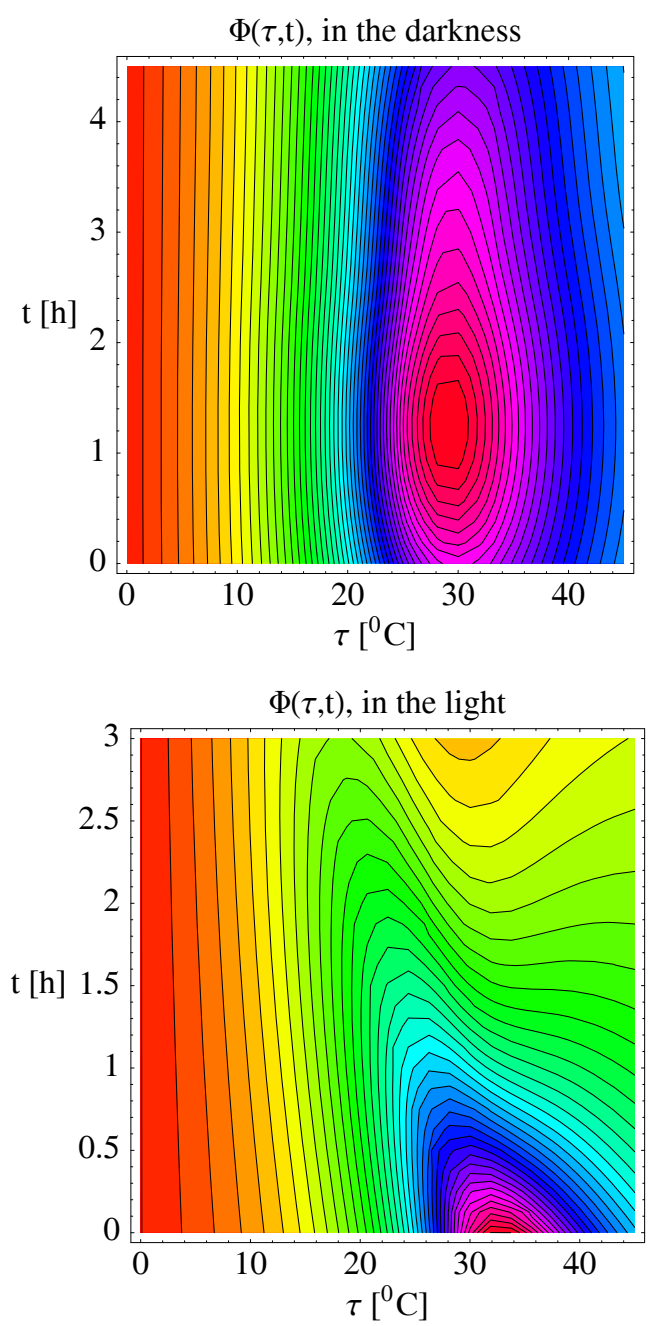

Figure 5. Contour plots (theory) of the calculated cell wall extensibility coefficient $\Phi(\tau, \mathrm{t})\left[\mathrm{cm}^{3} \mathrm{~J}^{-1} \mathrm{~h}^{-1}\right]$ for seedlings growing in the darkness and in the day light. In the case of exposure to the sun light, the crest of the optimum temperatures pronouncedly tends to the lower temperatures with increasing time, however, a remnant of high-temperature edge crest is also noticeable. causes the exponential decay. Moreover, in the case of application of the inhibitor an apparently interesting solution seen as "bifurcation" for times grater than approximately one hour appears. This means that after implementation of continuously added inhibitor the crest of optimum temperature in $\Phi(\tau, t)$ splits into two asymmetric branches.

In experiments performed in this study we were dealing with three-day-old seedlings of maize, however, the length of elongation zone of the coleoptiles was measured. In Fig. 5 we present the result of our model calculations for the cell wall extensibility coefficient of maize in the case of growth in darkness and exposure to the sun light. In the unperturbed experiment, $\Phi(\tau, t)$ initially increases and after reaching the maximum starts to decrease, though the changes are slight. A completely different picture results from our model calculations for the extensibility coefficient of maize coleoptiles exposed to the light (the right plots in Fig. 5). Both plots show clearly the strong influence of the external perturbation onto plant growth and consequently also onto the extensibility $\Phi$. The crest of optimum temperatures gains a curvature into the lower temperature ranges with increasing time. Moreover, a closer examination of the figure reveals a relict of a hightemperature edge crest. All contour plots have been obtained with the help of fitting results (Table 1).

Based on the continuity condition $\left[\Phi\left(\tau, t_{1}\right)\right]_{\mathrm{dark}}=$ $\left[\Phi\left(\tau, t_{1}\right)\right]_{\text {light }}$ at the time instant $t=t_{1}$ we have calculated next $3 \mathrm{D}$ plot (see Fig. 6) of $\Phi$ in function of time $t$ and temperature $\tau$ where the light, treated as an external perturbation, has been switched on at a certain time moment - here after $1.5 \mathrm{~h}$ from the beginning of experiment. The simulation reveals the slight increase of $\Phi(\tau, t)$ for the period of growth

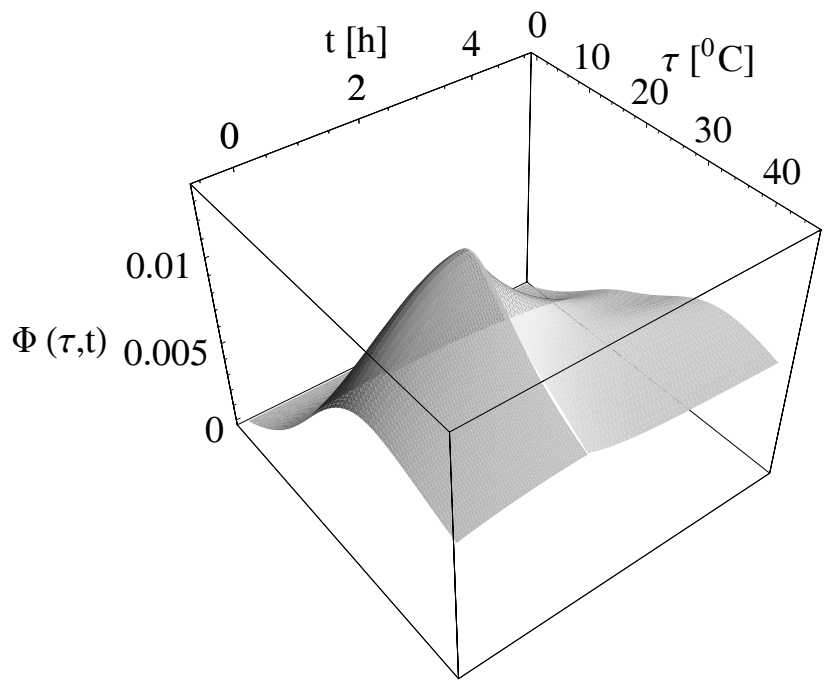

Figure 6. The calculated extensibility coefficient $\Phi(\tau, \mathrm{t})\left[\mathrm{cm}^{3} \mathrm{~J}^{-1} \mathrm{~h}^{-1}\right]$ in function of time $t$ and temperature $\tau$. A characteristic kink at the time $t=1.5 \mathrm{~h}$ at which the investigated seed has been exposed to the day light is clearly visible. 
Table 1: Simulation parameters $\phi_{0}, \alpha$ and $\tau^{*}$ obtained by fitting the Lorentz curve to the experimental data

\begin{tabular}{|c|c|c|c|c|}
\hline & after $1 / 2 \mathrm{~h}$ & after $1 \mathrm{~h}$ & after $3 / 2 \mathrm{~h}$ & after $2 \mathrm{~h}$ \\
\hline $\begin{array}{l}\text { Unperturbed case } \\
\text { maize coleoptile segments } \\
\text { elongation }[\mu \mathrm{m}]\end{array}$ & $\begin{array}{c}\phi_{0}=23 \pm 1 \\
\alpha=5.1 \pm 0.1 \\
\tau^{*}=28.8 \pm 0.3\end{array}$ & $\begin{array}{c}\phi_{0}=45 \pm 3 \\
\alpha=5.5 \pm 0.4\end{array}$ & $\begin{array}{c}\phi_{0}=69 \pm 2 \\
\alpha=5.7 \pm 0.3\end{array}$ & $\begin{array}{c}\phi_{0}=96 \pm 3 \\
\alpha=6.1 \pm 0.3\end{array}$ \\
\hline $\begin{array}{l}\text { Linear stimulation } \\
\text { maize coleoptile segments } \\
\text { elongation }[\mu \mathrm{m}]\end{array}$ & $\begin{array}{c}\phi_{0}=38 \pm 2 \\
\alpha=6.2 \pm 0.5 \\
\tau^{*}=28.7 \pm 0.4\end{array}$ & $\begin{array}{c}\phi_{0}=68 \pm 6 \\
\alpha=6.0 \pm 0.6\end{array}$ & $\begin{array}{l}\phi_{0}=100 \pm 5 \\
\alpha=5.8 \pm 0.6\end{array}$ & $\begin{array}{l}\phi_{0}=127 \pm 5 \\
\alpha=5.6 \pm 0.4\end{array}$ \\
\hline $\begin{array}{l}\text { Linear inhibition } \\
\text { maize coleoptile segments } \\
\text { elongation }[\mu \mathrm{m}]\end{array}$ & $\begin{aligned} \phi_{0} & =11.6 \pm 0.6 \\
\alpha & =3.9 \pm 0.4 \\
\tau^{*} & =28.8 \pm 0.4\end{aligned}$ & $\begin{aligned} \phi_{0} & =21.8 \pm 0.7 \\
\alpha & =4.1 \pm 0.2\end{aligned}$ & $\begin{aligned} \phi_{0} & =32.2 \pm 0.4 \\
\alpha & =4.3 \pm 0.3\end{aligned}$ & $\begin{array}{c}\phi_{0}=44 \pm 2 \\
\alpha=4.5 \pm 0.4\end{array}$ \\
\hline $\begin{array}{l}\text { Non-linear stimulation } \\
\text { maize coleoptile segments } \\
\text { elongation }[\mu \mathrm{m}]\end{array}$ & $\begin{aligned} \phi_{0} & =10.2 \pm 0.8 \\
\alpha & =4.9 \pm 0.5 \\
\tau^{*} & =29.1 \pm 0.3\end{aligned}$ & $\begin{aligned} \phi_{0} & =29.6 \pm 1.6 \\
\alpha & =5.0 \pm 0.5\end{aligned}$ & $\begin{array}{c}\phi_{0}=56 \pm 2 \\
\alpha=5.3 \pm 0.5\end{array}$ & $\begin{array}{l}\phi_{0}=103 \pm 7 \\
\alpha=5.8 \pm 0.6\end{array}$ \\
\hline $\begin{array}{l}\text { Non-linear inhibition } \\
\text { maize coleoptile segments } \\
\text { elongation }[\mu \mathrm{m}]\end{array}$ & $\begin{aligned} \phi_{0} & =10.2 \pm 0.7 \\
\alpha & =4.9 \pm 0.4 \\
\tau^{*} & =29.3 \pm 0.4\end{aligned}$ & $\begin{array}{c}\phi_{0}=22 \pm 2 \\
\alpha=6.4 \pm 0.4\end{array}$ & $\begin{array}{c}\phi_{0}=31 \pm 2 \\
\alpha=7.3 \pm 0.4\end{array}$ & $\begin{array}{c}\phi_{0}=33 \pm 3 \\
\alpha=7.6 \pm 0.4\end{array}$ \\
\hline $\begin{array}{l}\text { „Dark“ case } \\
\text { maize whole coleoptiles } \\
\text { elongation }[\mathrm{mm}]\end{array}$ & $\begin{array}{c}\phi_{0}=0.038 \pm 0.002 \\
\alpha=9.0 \pm 0.6 \\
\tau^{\star}=27.1 \pm 0.2\end{array}$ & $\begin{array}{c}\phi_{0}=0.070 \pm 0.004 \\
\alpha=8.4 \pm 0.5\end{array}$ & $\begin{array}{c}\phi_{0}=0.092 \pm 0.004 \\
\alpha=8.2 \pm 0.5\end{array}$ & $\begin{array}{c}\phi_{0}=0.116 \pm 0.006 \\
\alpha=8.1 \pm 0.5\end{array}$ \\
\hline $\begin{array}{l}\text { "Light" case } \\
\text { maize whole coleoptiles } \\
\text { elongation }[\mathrm{mm}]\end{array}$ & $\begin{array}{c}\phi_{0}=0.013 \pm 0.001 \\
\alpha=6.6 \pm 0.5 \\
\tau^{\star}=29.3 \pm 0.3\end{array}$ & $\begin{array}{c}\phi_{0}=0.027 \pm 0.002 \\
\alpha=7.6 \pm 0.6 \\
\tau^{*}=28.6 \pm 0.4\end{array}$ & $\begin{array}{c}\phi_{0}=0.042 \pm 0.002 \\
\alpha=8.3 \pm 0.6 \\
\tau^{\star}=26.6 \pm 0.4\end{array}$ & $\begin{array}{c}\phi_{0}=0.058 \pm 0.003 \\
\alpha=8.9 \pm 0.7 \\
\tau^{*}=25.5 \pm 0.4\end{array}$ \\
\hline
\end{tabular}

$\phi_{0}$ and $\alpha$, the height and the width of the Lorentz-like curve, respectively; $\tau^{*}$, the optimum (critical) temperature of growth.

in the darkness (which is equivalent to the control) and the rapid exponential decay after exposure to the light. It is a very interesting fact that the response to the light occurs almost immediately (without noticeable retardation), right after putting the light on.

Combination of Figs. 5 and 6 give rise to the following interpretation. Since turning the light on causes the exponential damping of the $\Phi$ coefficient at optimum temperatures (as it is seen in Fig. 6) the growing young maize must undergo some internal (biochemical) processes leading to the strong inhibition of the growth rate. Indeed, we encounter such situation in Fig. 5. Also, due to the absorption of energy for directed morphogenesis, and scattering (dissipation) of the light quanta, the internal raise of temperature inside the plant cell provokes the observed lowering of the optimum temperature crest in the direction of ascending time.

\section{Discussion}

In this paper, we develop a simple but efficient physical model of temperature dependence of plant cell growth. This model, hereafter designed as a "generalized Lockhart equation" (GLE), goes much further compared to its original form proposed by Lockhart (1965a,b), since the environmental temperature has been included. Moreover, by constructing our model equation as temperature dependent, we proceed next to involve into GLE all possible external physical and (bio)chemical factors influencing plant cell growth. Among others, it accounts for the incident light (which investigation was of our main concern in this paper) and growth stimulators/inhibitors. Indeed, as we have mentioned in the introductory part of this paper, already the early studies concluded that radiant energy causes an inhibition in the rate of elongation and that light retards not only the rate of stem elongation but also causes reduction in the plastic extensibility of the cell walls. However, this is also the central point of this study: the inclusion of the black body radiation term into GLE (representing the interaction between the light quanta and photoreceptors) may successfully report on transient changes in plant cells when exposed to the light. Also the above mentioned reduction in extensibility can be described by GLE when proper terms are considered. Having in mind that the last two terms in Eq. (1), responsible for the light and external pressure, can be treated simultaneously - a suitable experiment can be projected and the outcoming data when introduced to our model can report on the mechanical properties of the cell wall (by calculating $\Phi(\tau, t)$ ). To sum up, we believe that GLE, when properly applied ( via the Lorentz-like fit) to the empirical (elongation) data obtained 
by plant physiologists, can serve as a new versatile research tool which, in particular, can (indirectly) yield a theoretical insight into the problems of plant cell growth, also in the context of cell wall extensibility.

We are very well aware of the limitations of the equations we develop. Surely we are confined to the 'expansion' component of growth and we do not refer to the cell proliferation, however, this is a consequence of the fact that we go along the path proposed by Lockhart (1965a,b) is his equations. Also, the validity of our equation is limited to the non-dissipative temperature region (no membrane leakage) where the Lockhart equation holds. Even though the biochemistry is not discussed in this paper, it is implicit in the phenomenological functions which account for the effects like enzymes activity, protein synthesis, cell respiration and biomass production. At this early stage of investigation, though, it would be useful to have an analytical model and accordingly - a computable description of cell stretching that correctly accounts for the way it depends on external factors such as light and/or phytohormones in the thermodynamical environment. Moreover, it is known that $\Phi$ is essentially the inverse of viscosity and that some hormones, like auxin, influence on viscosity. Therefore, light and plant hormones might affect only $\Phi$ instead of $P-Y$.

\section{Appendix}

The energy of irradiation $\Delta E$ as introduced in the main part of the paper by the integral over Planck's distribution and over the whole optical spectrum (from $400 \mathrm{~nm}$ to 700 $\mathrm{nm}$, hereafter denoted as [OR] (optical range)) - may be considered as beneath.

Taking into account red/far red spectrum of the phytochrome family actively contributing to the retardation of the cell/organ extension the expression for $\Delta E$ in $V$ units takes on the form

$$
\begin{aligned}
\frac{\Delta E}{V} & =\int_{[\mathrm{OR}]} \frac{8 \pi h}{c^{3}} \frac{v^{3}}{e^{\frac{h v}{k_{B} T}}-1} d v \cong \int_{[\mathrm{OR}]} \frac{8 \pi h}{c^{3}}\left(\sum_{\alpha} \delta\left(v-v_{\alpha}\right)\right) \frac{v^{3}}{e^{\frac{h v}{k_{B} T}}-1} d v \\
& =\sum_{\alpha} \frac{8 \pi h}{c^{3}} \int_{[\mathrm{OR}]}\left(\delta\left(v-v_{\alpha}\right)\right) \frac{v^{3}}{e^{\frac{h v}{k_{B} T}}-1} d v=\frac{8 \pi h}{c^{3}} \sum_{\alpha} \frac{v_{\alpha}^{3}}{e^{\frac{h v_{\alpha}}{k_{B} T}}-1}(17)
\end{aligned}
$$

where $a \in\{R, F R\}$ denotes the red (R) or far red (FR) fragment of spectrum for the photoreceptor. The Dirac's delta function $\delta\left(v-v_{\alpha}\right)$ cuts out a desired frequency $v_{\alpha}$ from the continuous range. Thus

$$
\frac{\Delta E}{V}=\frac{8 \pi h}{c^{3}}\left(\frac{v_{R}^{3}}{e^{\frac{h v_{R}}{k_{B} T}}-1}+\frac{v_{F R}^{3}}{e^{\frac{h v_{F R}}{k_{B} T}}-1}\right)
$$

where $\Delta E$ is the total amount of energy absorbed by the phytochromes in the plant cell/organ. However, the absorption efficiency $\eta$ is also temperature dependent and can be identified with the dark / light ratio, Fig. 3. In this study the $\eta$ parameter has been obtained by the normalization of this ratio such that $\eta\left(\tau_{\text {opt }}\right)=1$.

\section{References}

Arteca R. (1996): Plant Growth Substances: Principles and Applications. Chapman \& Hall, New York

Bandurski R. S., Cohen J. D., Slovin J., Reinecke D. M. (1995): Auxin biosynthesis and metabolism. In: Plant Hormones: Physiology, Biochemistry and Molecular Biology. pp. 39-65, Kluwer Academic Press, Dordrecht

Cao W. H., Liu J., He X. J., Mu R. L., Zhou H. L., Chen S. Y., Zhang J. S. (2007): Modulation of ethylene responses affects plant salt-stress responses. Plant Physiol. 143, 707-719; doi:10.1104/pp.106.094292

Casal J. J., Sanchez R. A., Botto J. F. (1998): Modes of action of phytochromes. J. Exp. Bot. 49, 127-138; doi:10.1093/ jexbot/49.319.127

Christie J. M., Briggs W. R. (2001): Blue light sensing in higher plants. J. Biol. Chem. 276, 11457-11460; doi:10.1074/jbc. R100004200

Cleland R. E (1986): The role of hormones in wall loosening and plant growth. Aust. J. Plant Physiol. 13, 93-103

Cosgrove D. J. (1986): Biophysical control of plant cell growth. Annu. Rev. Plant Physiol. 37, 377-405; doi:10.1146/annurev.pp.37.060186.002113

Cosgrove D. J. (1993): How do plant cell walls extend? Plant Physiol. 102, $1-6$

De Greef J. A. (1996): Phytochrome research in whole plant physiology. Wild-type seedlings versus mutants and transgenic plants. Physiol. Plant. 98, 377-380; doi:10.1034/j.13993054.1996.980220.x

Deng X. W., Quail P. H. (1999): Signalling in light-controlled development. Sem. Cell Dev. Biol. 10, 121-129; doi:10.1006/ scdb.1999.0287

Edelmann H. G. (1995): Wall extensibility during hypocotyl growth: a hypothesis to explain elastic-induced wall loosening. Physiol. Plant 95, 296-303; doi:10.1111/j.1399-3054.1995. tb00841.x

Fogg G. E. (1975): The growth of plants. (Ed. Clay Richard), The Chaucer Press, Bungay-Suffolk

Friml J. (2003): Auxin transport - shaping the plant. Curr. Opinion Plant Biol. 6, 7-12, http://www.uni-tuebingen.de/uni/bee/ friml; doi:10.1016/S1369526602000031

Hoagland D. R., Arnon D. J. (1950): The water culture method for growing plants without soil. Calif. Agric. Exp. Stat. Circ. 347, 1-32

Ikeda T., Nonami H., Fukuyama T., Hashimoto Y. (1999): Hydraulic contribution in cell elongation of tissue-cultured plants: growth retardation induced by osmotic and temperature stresses and addition of 2,4-dichlorophenoxyacetic acid and benzylaminopurine. Plant 
Cell Environm. 22, 899-912; doi:10.1046/j.13653040.1999.00463.x

Koller D. (1990): Light-driven leaf movements. Plant Cell Environm. 13, 615-632; doi:10.1111/j.1365-3040.1990. tb01079.x

Kutschera U. (2000): Cell expansion in plant development. Rev. Bras. Fisiol. Veg. 12, 65-95

Lewicka S. (2006): General and analytic solutions of the Ortega equation. Plant Physiol. 142, 1346-1349; doi:10.1104/ pp.106.086751

Lewicka S., Pietruszka M. (2006): Theoretical search for the growthtemperature relationship in plants. Gen. Physiol. Biophys. 25, 125-136

Lewicka S., Pietruszka M. (2007): Anisotropic plant cell elongation due to ortho-gravitropism. J. Math. Biol. 54, 91-100; doi:10.1007/s00285-006-0049-3

Lockhart J. A. (1965a): An analysis of irreversible plant cell elongation. J. Theor. Biol. 8, 64-75; doi:10.1016/00225193(65)90077-9

Lockhart J. A. (1965b): Cell extension. In: Plant Biochemistry. (Eds. Bonner J., Varner J. E.), pp. 826-849, Academic Press, New York-London

Madlung A., Behringer F., Lomax T. (1999): Ethylene plays multiple nonprimary roles in modulating the gravitropic response in tomato. Plant Physiol. 120, 897-906; doi:10.1104/ pp.120.3.897

Montague M. J. (1997): Exogenous jasmonic and abscisic acids act differentially in elongating tissues from oat stem segments. J. Plant Growth Regul. 16, 11-19; doi:10.1007/PL00006969

Nakamura Y., Wakabayashi K., Kamisaka S., Takayuki H. (2002): Effects of temperature on the cell wall and osmotic properties in dark-grown rice and azuki bean seedlings. J. Plant Res. 115, 455-461; doi:10.1007/s10265-002-0058-2

Nakasako M., Iwata T., Inoue K., Takutomi S. (2005): Light-induced global structural changes in phytochrome A regulating photomorphogenesis in plants. FEBS J. 272, 603-612; doi:10.1111/j.1742-4658.2004.04508.x

Nikolić R., Mitić N., Miletić R., Nešković M. (2006): Effects of cytokinins on in vitro seed germination and early seedling morphogenesis in Lotus corniculatus L. J. Plant Growth Regul. 25, 187-194; doi:10.1007/s00344-005-0129-4

Pietruszka M., Lewicka S., Pazurkiewicz-Kocot K. (2006): Thermodynamics of time-irreversible plant cell growth. Acta Soc. Bot. Pol. 75, 183-190

Pietruszka M., Lewicka S., Pazurkiewicz-Kocot K. (2007): Temperature and the growth of plant cells. J. Plant Growth Regul. 26, 15-25; doi:10.1007/s00344-006-0023-8
Pietruszka M., Lewicka S. (2007a): Anisotropic plant growth due to phototropism. J. Math. Biol. 54, 45-55; doi:10.1007/ s00285-006-0045-7

Pietruszka M., Lewicka S. (2007b): Effect of temperature on plant elongation and cell wall extensibility. Gen. Physiol. Biophys. 26, 40-47

Proseus T. E., Zhu G., Boyer J. S. (2000): Turgor, temperature, and the growth of plant cells: using Chara corallina as a model system. J. Exp. Bot. 51, 1481-1494; doi:10.1093/ jexbot/51.350.1481

Rayle D. L., Cleland R.E. (1992): The Acid Growth Theory of auxininduced cell elongation is alive and well. Plant Physiol. 99, 1271-1274; doi:10.1104/pp.99.4.1271

Reinhardt D., Pesce E. R., Stieger P., Mandel T., Baltensperger K., Bennett M., Traas J., Friml J., Kuhlemeier C. (2003): Regulation of phyllotaxis by polar auxin transport. Nature 426, 255-260; doi:10.1038/nature02081

Ross J. J., O’Neill D. P., Rathbone D. A. (2003): Auxin-Gibberellin interactions in pea: integrating the old with the new. J. Plant Growth Regul. 22, 99-108; doi:10.1007/s00344003-0021-z

Scott T. K., Sievers A., Zieschang H. E., Wayne R. (1992): Forum: what remains of the Cholodny-Went theory? Plant Cell Environm. 15, 759-794

Smith H. (2000): Phytohormones and light signal perception by plants - an emerging synthesis. Nature 407, 585-591; doi: $10.1038 / 35036500$

Stanley H. (1971): Introduction to Phase Transitions and Critical Phenomena. Oxford University Press

Trewavas A. J. (1991): How do plant growth substances work? Plant Cell. Environm. 14, 1-12; doi:10.1111/j.1365-3040.1991. tb01366.x

Tsai F. Y., Lin C. C., Kao C. H. (1997): A comparative study of the effects of abscisic acid and methyl jasmonate on seedling growth of rice. Plant Growth Regul. 21, 37-42; doi:10.1023/A:1005761804191

Went F. W., Thimann K. V. (1937): Phytohormones. Macmillan, New York

Wright S. T. C. (1966): Growth and cellular differentiation in the wheat coleoptile (Triticum vulgare). Factors influencing the growth responce to gibberelic acid, kinetin and indole-3-acetic acid. J. Exp. Bot. 17, 165-176; doi:10.1093/ jxb/17.1.165

Received: May 5, 2009

Final version accepted: November 24, 2009 